\section{Stentthrombosen nach Implantation von Stents zur Therapie des PTS häufig}

Sebastian T et al. Incidence of Stent Thrombosis after Endovascular Treatment of Iliofemoral or Caval Veins in Patients with the Postthrombotic Syndrome. Thromb Haemost 2019; 119: 2064-2073

Grundpfeiler der Behandlung der tiefen Venenthrombose (TVT) ist die antikoagulative Therapie (AT). Die Entstehung des postthrombotischen Syndroms (PTS) scheint nur ihre Qualität und nicht ihre Dauer zu beeinflussen. Patienten/-innen mit PTS, denen Stents implantiert wurden, tragen das Risiko von Stentthrombosen. Inzidenz und Risikofaktoren von Stentthrombosen mit oder ohne AT sind kaum bekannt. Dieser Fragestellung ging die Studie nach.

Die Daten für ihre Analyse entnahm die Arbeitsgruppe dem prospektiv geführten Schweizer Register venöser Stents (Swiss Venous Stent Registry). Aus diesem identifizierten sie 136 Patienten/-innen mit PTS im medianen Alter von 43 (IQR 31-56) Jahren. Bei allen bestand anamnestisch eine TVT der V. cava inferior oder iliakaler Venen. Wegen postthrombotischer Okklusion mindestens eines dieser Gefäße war bei ihnen in den Universitätskliniken Bern oder Zürich eine endovaskuläre Rekonstruktion mit Stent-Implantation erfolgt. Der mediane Zeitabstand zwischen erster TVT und der Intervention betrug 4,5 (IQR 1-21) Jahre. Die Diagnose einer Stentthrombose wurde anhand von Duplexsonografien oder Venografien gestellt. Für ihre Analyse unterteilten Sebastian et al. die Patienten/-innen in 2 Gruppen:

Gruppe 1 mit weitergeführter AT während des gesamten Follow-ups ( $n=93 ; 68 \%)$

Gruppe 2 mit unterbrochener AT, bei der während des Follow-ups die AT abgebrochen oder auf eine prophylaktische Dosis reduziert wurde $(n=43 ; 32 \%)$.

Die Patienten/-innen der Gruppe 1 wiesen höhere Symptom-Scores (rVCSS - revised Venous Clinical Severity Score, Villalta Score) bei den Ausgangswerten auf. Sie waren häufiger fettleibig und hatten anamnestisch häufiger rezidivierende venöse Thromboembolien. Sie erhielten mehr Stents implantiert, die häufiger in die $\mathrm{V}$. cava inferior implantiert wurden als in Gruppe 2. Die Patienten/-innen in Gruppe 2 waren jünger, doppelt so häufig Frauen und wiesen häufiger ein May-ThurnerSyndrom auf.

Das mediane Follow-up betrug 20 (IQR 940) Monate. Die AT in Gruppe 2 wurde nach einer medianen Dauer von 12 (IQR 6-14) Monaten abgebrochen. Stentthrombosen traten nach einem Median von 96 (IQR 15-366) Tagen auf, in Gruppe 1 waren es 89 (IQR 13-136) Tage und 289 (IQR 69-900) Tage in Gruppe 2. Die kumulative Inzidenz von Stentthrombosen in der gesamten Kohorte betrug während der ersten 6 Monate 13,7\% (95\%-KI: 7,8$19,6 \%$ ). Innerhalb von 36 Monaten betrug sie $21,2 \%$ (95\%-KI: 13,2-29,2\%). Die Autoren/-innen ermittelten eine zeitadjustierte Inzidenzrate von 11,2 Ereignissen/
100 Patientenjahre (95\%-KI: 7,7-16,2). Für die Zeit mit AT ermittelten sie 11,3 (95\%-KI: 7,3-17,3), für die Zeit ohne AT 11,2 (95\%-KI: 5,3-23,6) Ereignisse/ 100 Patientenjahre. Das May-Thurner-Syndrom war mit einer erniedrigten Inzidenz von Stentthrombosen (Hazard Ratio [HR]: 0,37; $95 \%$-KI: 0,15-0,91) assoziiert. Mit einer höheren Inzidenz waren assoziiert:

- ein Lebensalter<40 Jahren (HR: 2,6; 95\%-KI: 1,03-4,94);

- Stents unterhalb der V. femoralis communis (HR: 3,03; $95 \%-K I: ~ 1,28-7,19)$ sowie

- postthrombotische Zustromvenen (HR: 2,92; $95 \%-K I: ~ 1,36-6,25)$.

Beim letzten Follow-up waren 90 Patienten/-innen (66\%) frei von einem PTS.

\section{FAZIT}

Laut den Autoren/-innen war die Inzidenzrate von Stentthrombosen innerhalb von 6 Monaten beträchtlich hoch mit einer persistierenden jährlichen Inzidenzrate von 4,1\% und 3,4\%. Die Inzidenzraten für die Zeiten mit und ohne AT sollten vorsichtig interpretiert werden. Das optimale Antikoagulans und die optimale Therapiedauer für PTS-Patienten/-innen mit venösen Stents müssten in weiteren randomisierten Studien erforscht werden.

Dr. Gabriele Dobler, Berlin 